\title{
Arsenite Activation of PI3K/AKT Cell Survival Pathway is Mediated by $p 38$ in Cultured Human Keratinocytes
}

\author{
Kailene Souza,* David Audoen Maddock, Quanshun Zhang,* Jianping Chen, Cheuk Chiu, Shashi \\ Mehta, and Yinsheng Wan
}

Department of Biology, Providence College, and Roger Williams Medical Center, Providence, RI, USA

Accepted September 24, 2001

\begin{abstract}
Background: Arsenic has been considered as a carcinogen. Recently the issue of arsenic in drinking water raised an unprecedented social concern on human health, and yet the molecular mechanisms through which arsenic induces cancer remain unknown. Activation of cell survival pathway leading to the activation of eNOS has been associated with various types of cancer. The objective of this study was to investigate the pathway leading to the activation of eNOS in response to arsenite using human keratinocytes.

Materials and Methods: Cultured keratinocytes (HaCat cells) were exposed to arsenite with or without pretreatment of various inhibitors. Western blot analysis was performed to determine the activation of p38, AKT, eNOS. EGFR tyrosine phosphorylation was detected by immunoprecipitation and Western blot analysis. pNPP assay was used to measure phosphatase activity in cell lysate. FACS
\end{abstract}

analysis was performed for the determination of generation of reactive oxygen species.

Results: Arsenite induced the activation of AKT at both Ser473 and Thr308, and its downstream effector eNOS in cultured human keratinocytes. Arsenite also induced phosphorylation of p38. PI-3-kinase inhibitors, Wortmannin and LY294002 inhibited arsenite-induced phosphorylation of AKT and eNOS but had no effect on phosphorylation of p38. Interestingly, however, SB203580, a known p38 inhibitor, completely inhibited arsenite-induced phosphorylation of AKT and eNOS. Arsenite induced generation of reactive oxygen species and inactivated phosphatase activity, but did not activate EGF receptor tyrosine phosphorylation. Conclusions: Collectively, our data indicate that arsenite induces activation of AKT and eNOS, via PI-3-kinase and p38 pathway, likely bypassing the activation of EGF receptor in cultured human keratinocytes.

\section{Introduction}

Arsenite, alternatively referred to as arsenic, exists in soil, water, air, and even in living organisms. It is a carcinogen associated with cancers of epithelial origin such as skin, lung, liver, and bladder cancer (1). Arsenite has recently raised unprecedented social concern due to unwanted environmental pollution, especially in drinking water (2). While it has been recognized that ingesting even trace amounts of environmental arsenite poses serious health risks, the molecular mechanism by which arsenite causes melanomas or epithelial cancers remains relatively unexplored. It has been observed that arsenite produces sustained alterations in the growth characteristics of human fibroblasts, and then postulated that the proliferationenhancing effect of arsenic on cells contributes to its ability to cause cancer (3). It has been also

Send correspondence and reprint requests to: Yinsheng Wan, Department of Biology, Providence College, 549 River Avenue, Providence, RI, 02918, USA. Phone: (401)-865-2507; fax: (401)-865-1438; e-mail: yswan@providence.edu.

*Present Address: (KS) Department of Hematology/Oncology, Beth Israel Deaconess Medical Center, Harvard Medical School 330 Brookline Ave, Boston, MA, 02215, USA; (QZ) Department of Biomedical Sciences, Tufts University School of Veterinary Medicine, North Grafton, MA, 01536, USA. shown that arsenite reduces p53 levels, while concomitantly increasing the p53 regulatory protein mdm2 levels. It is suggested that this disruption of the $\mathrm{p} 53-\mathrm{mdm} 2$ loop, instrumental to the regulation of cell cycle arrest, may offer another model for arsenite-related skin carcinogenesis (4). Ultimately, however, the question of how arsenite enhances cell proliferation has not yet been answered. In addition, the pathways leading to the disruption of the p53/mdm2 loop have not been unraveled. A recent study suggests that activation of AKT provides a critical cell survival signal required for tumor progression (5). Our previous data that the PI3K/AKT pathway contributes to UV-induced skin cancer supports the above notion (6). Activation of AKT by PI3K results in inhibition of apoptotic signals and promotion of cell survival signals (7). Abnormal survival of mutated cells could further favor cancer formation and progression. To gain insight into the understanding of molecular mechanisms of arseniteinduced human skin cancer, we investigated the pathway leading to the activation in cultured human keratinocytes in response to arsenite exposure. We demonstrate in this study that arsenite activates the PI3K/AKT cell survival pathway in cultured human keratinocytes, and that the arsenite-induced activation of AKT and eNOS is mediated by $\mathrm{p} 38$ MAP kinase. 


\section{Materials and Methods Cell Culture}

Human keratinocytes (HaCat cell line) were cultured as described previously (6). Cells were grown as monolayer cultures in DMEM medium with $10 \%$ FBS in a humidified incubator with $5 \% \mathrm{CO}_{2}$ at $37^{\circ} \mathrm{C}$. Medium was usually deprived of serum for overnight prior to reagent treatment.

\section{FACS Analysis}

Cultured human keratinocytes were deprived of serum overnight and loaded with $1 \mu \mathrm{M}$ of a fluorescent dye, dihydrorhodamine (DHR) that reacts with ROS in cells and results in a change of fluorescence. Cells were treated with arsenite (sodium arsenite, $200 \mu \mathrm{M}$ ) for various time points. The changes in fluorescence in arsenite-irradiated cells were quantified by FACS analysis as described previously (8). Induction of ROS generation was expressed in arbitrary units.

\section{EGF Receptor Tyrosine Phosphorylation}

Reagents-treated keratinocytes were collected. Total and tyrosine-phosphorylated EGF receptors in whole cell extracts of cultured keratinocytes were determined by immunoprecipitation and Western blot analysis, using antibodies to EGF receptors (Santa Cruz Biotechnologies), and phosphortyrosine (PY-20, Transduction Laboratories). Immunoprecipitated proteins were visualized by enhanced chemiluminescence detection (ECL), and quantified by laser densitometry, as described previously (6).

\section{Phosphorylation of $p 38, A K T$, and eNOS}

Cultured keratinocytes after treatment with various reagents were subjected to protein extraction, and electrophoretically separated proteins were transferred to Immobilon-P membranes. The membranes were blocked with $5 \%$ dry milk in TBS containing $0.05 \%$ Tween-20 (TBST) for at least one hour at room temperature. After blocking, the membranes were incubated with specific antibodies against AKT, or phospho-AKT, p38, or phospho-p38, eNOS or phospho-eNOS (New England Biolabs) at appropriate dilutions. The blots were incubated with horseradish peroxidase-conjugated anti-rabbit IgG (1:5000, Amersham) for $1 \mathrm{~h}$. Antibody binding was detected by using the enhanced chemiluminescence (ECL) detection system (Amersham).

\section{Phosphatase Assay}

Cultured keratinocytes were treated with arsenite (200 $\mu \mathrm{M})$ for various time points. After treatment, the medium was removed and $400 \mu$ l of cell lysis buffer (RIPA buffer without sodium vanadate, with protease inhibitor cocktail) was added to the culture dish. The cell extracts were scraped out of the dish and transferred to the microfuge tube. After centrifugation at $14000 \mathrm{rpm}$ for $10 \mathrm{~min}$, supernatants were used for phosphatase assay. Briefly, to the wells of a microtiter plate, $100 \mu \mathrm{l}$ of PNPP solution $(20 \mathrm{mg}$ of p-nitrophenyl phosphate in $5 \mathrm{ml}$ assay buffer containing $50 \mathrm{mM}$ Tris, pH 7.6, $100 \mathrm{mM} \mathrm{NaCl}, 0.1 \mathrm{mg} / \mathrm{ml}$ BSA) and $50 \mu \mathrm{l}$ of cell lysate were added. The plate with reaction mixture was incubated at $37^{\circ} \mathrm{C}$; yellow color developed as the reaction proceeded. $100 \mu \mathrm{l}$ of $13 \% \mathrm{~K}_{2} \mathrm{HPO}_{4}$ was added to stop the reaction, and OD405nm was measured in ELISA plate reader.

\section{Results}

Arsenite and $\mathrm{H}_{2} \mathrm{O}_{2}$ Induce Phosphorylation of $p 38, A K T$ and eNOS in Cultured Human Keratinocytes

Existing data almost exclusively indicate that arsenite induces apoptosis or cell death $(9,10)$. Our previous study demonstrated that extracellular insults such as UV irradiation induces PI3-kinase/AKT cell survival pathway in cultured human keratinocytes in vitro and in human skin in vivo (6). These data support the notion that cells fight against extracellular insults through promoting both cell survival and cell suicide in a kind of Ying-Yang balance. To test whether arsenite activates AKT in cultured human keratinocytes, we treated cells with arsenite and measured the phosphorylation of AKT at residues Ser473 and Thr308. The results showed that AKT phosphorylation started at $15 \mathrm{~min}$ post arsenite treatment, peaked at about $1 \mathrm{~h}$ and remained elevated up to two hours, while the total amount of AKT remained unchanged when the cells were exposed to arsenite (Fig. la-A). As with AKT, arsenite treatment did not change the level of total p38, however, it also induced the phosphorylation of p38 in a time dependent manner (Fig. la-B). We also found that $\mathrm{H}_{2} \mathrm{O}_{2}$ weakly induced AKT phosphorylation in a pattern similar to that of arsenite. $\mathrm{H}_{2} \mathrm{O}_{2}$ treatment showed a similar pattern in inducing the phosphorylation of $\mathrm{p} 38$. It has been previously shown that one of the downstream effectors of AKT in PI3K/AKT pathway is eNOS (11). Since we had observed that arsenite induced the phosphorylation of AKT, we next investigated whether arsenite activates downstream effector eNOS. Westernblot analysis, using anti-phospho-eNOS antibody, revealed that arsenite did in fact induce the phosphorylation of eNOS (Fig. la-C). The phosphorylation started $30 \mathrm{~min}$ after arsenite treatment and peaked at about 2 hours. The quantitative data of arsenite-induced activation of p38, AKT and eNOS were shown in Fig. 1b. Likewise $\mathrm{H}_{2} \mathrm{O}_{2}$ induced the phosphorylation of eNOS, and in contrast to arsenite quite strongly. The total eNOS remains unaltered post treatment.

Arsenite-induced Activation AKT and eNOS is not Mediated by Antioxidant Sensitive Pathway but Mediated by $\mathrm{PI} 3 \mathrm{~K}$ and $\mathrm{p} 38$

To examine the role of ROS in the arsenite-induced phosphorylation of p38 and AKT, we used antioxidants NAC (N-acetyl-L-cysteine) and PDTC (pyrrolidinedithiocarbamate), and NADPH oxidase 
a

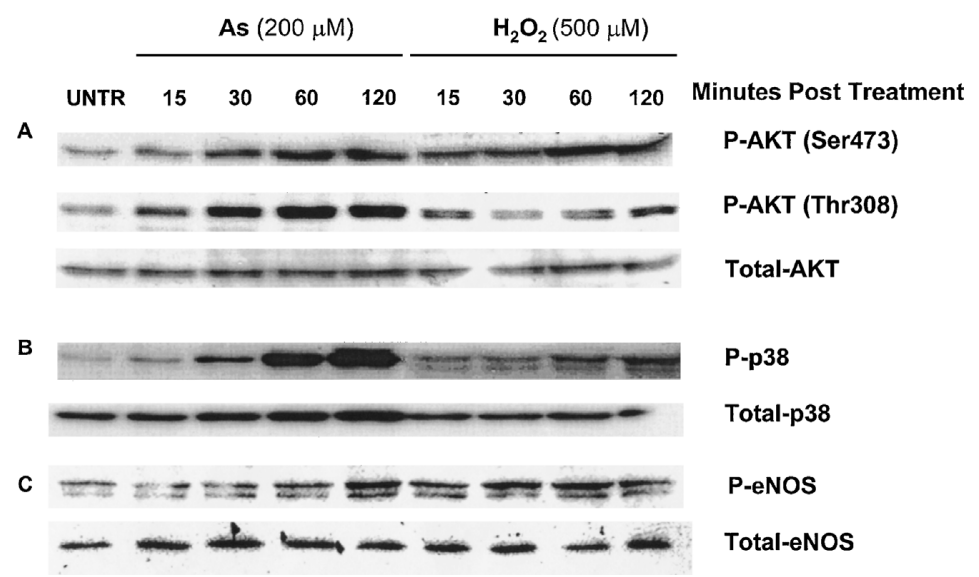

b

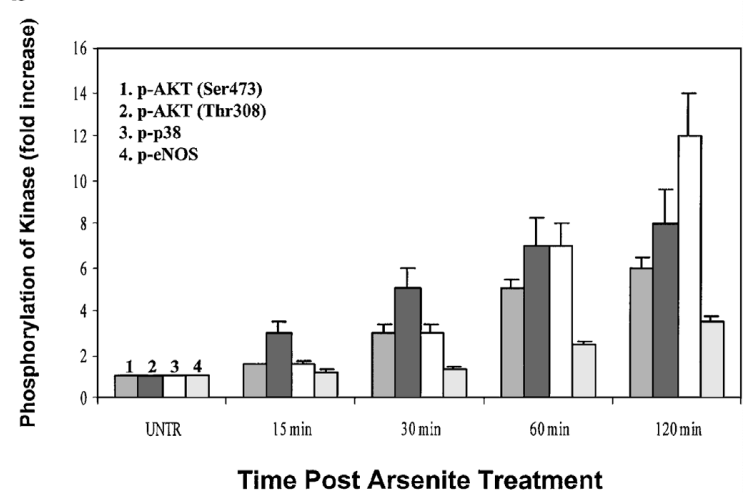

Fig. 1. Arsenite and $\mathrm{H}_{2} \mathrm{O}_{2}$ induce phosphorylation of p38, AKT and eNOS in cultured human keratinocytes. (a) Cultured human keratinocytes were treated with arsenite $(200 \mu \mathrm{M})$ and $\mathrm{H}_{2} \mathrm{O}_{2}$, and cells were lysed at various time points. Protein extracts were subjected to SDS-PAGE and Westernblot analysis for phosphorylation of p38 or AKT or eNOS with anti-phospho-p38 or antiphospho-AKT or anti-phospho-eNOS antibody respectively. Total p38, or AKT or eNOS was determined by stripping the membranes and re-probing with anti-p38, or anti-AKT, or anti-eNOS antibody. (b) Quantitative densitometric data for the phosphorylation p38, AKT and eNOS after arsenite treatment. The results from three independent experiments were analyzed and expressed as fold increase (mean $\pm \mathrm{SD}, \mathrm{n}=3$ ).

inhibitor, DPI (diphenylene iodonium chloride). The results showed that while arsenite expectedly induced phosphorylation of $\mathrm{p} 38$ and AKT, pretreatment of the cells with ROS scavengers NAC or PDTC did not inhibit either arsenite-induced phosphorylation of p38 or AKT or eNOS (Fig. 2). DPI had no effect on arsenite-induced p38 and AKT phosphorylation in cultured human keratinocytes. These data surprisingly but obviously indicate that arsenite-induced p38 and AKT activation are not mediated by anti-oxidant sensitive pathway in cultured human keratinocytes. To examine the role of PI 3-kinase or p38 in the arseniteinduced activation of AKT and eNOS, we used LY294002 and Wortmannin, two well-characterized PI 3-kinase inhibitors, and SB203580, a p38 inhibitor. Pretreatment of cells with LY294002 or Wortmannin dramatically inhibited arsenite-induced phosphorylation of both AKT and eNOS. As has been demonstrated for growth factors (12), PI 3-kinase appears to be involved in the activation of AKT post arsenite exposure. As expected, pretreatment with SB203580 inhibited arsenite-induced phosphorylation of $\mathrm{p} 38$. Interestingly, however, SB203580 almost completely inhibited arsenite-induced phosphorylation of AKT and eNOS. These data suggest that arsenite-induced activation of AKT and eNOS is mediated by p38 pathway and p38 is upstream of PI3K.

\section{Arsenite Induces ROS Generation and Inactivates \\ Phosphatase but does not Induce EGF Receptor Activation in Cultured Human Keratinocytes}

Reactive oxygen species (ROS) are involved in various signaling pathways (13). Our above data seemed to suggest that reactive oxygen species might be involved in arsenite-induced phosphorylation of p38 and AKT (Fig. 1). For this reason we examined whether arsenite induces generation of reactive oxygen species in cultured human keratinocytes. FACS analysis showed that arsenite did stimulate ROS generation in a time dependent manner (Fig. 3A).

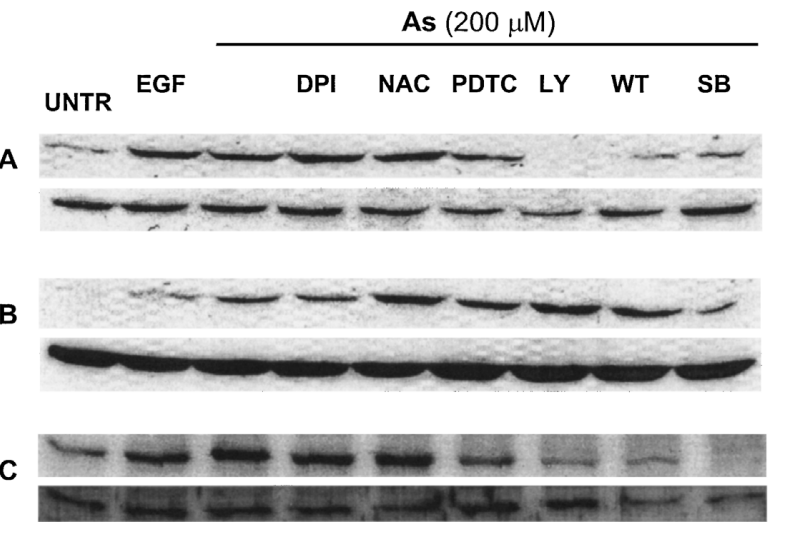

Pretreatment

P-AKT (Ser473)

Total-AKT

P-p38

Total-p38

P-eNOS

Total-eNOS
Fig. 2. Arsenite-induced activation AKT and eNOS is not mediated by antioxidant sensitive pathway but is mediated by PI3K and p38. Cultured human keratinocytes were pretreated with DPI $(10 \mu \mathrm{M})$, or NAC $(5 \mathrm{mM})$, or PDTC $(10 \mu \mathrm{M})$, or LY $(10 \mu \mathrm{M})$, WT $(10 \mu \mathrm{M})$ for 2 hours, then treated with arsenite $(200 \mu \mathrm{M})$ for 1 hour for analysis of $\mathrm{p} 38$ and AKT, and 2 hours for analysis of eNOS. Cells were lysed in RIPA buffer, and proteins were subjected to SDS-PAGE and Western blot analysis for phosphorylation of p38 or AKT with anti-phospho-p38 or anti-phospho-AKT or phospho-eNOS antibody, or for total p38 or AKT with anti-p38 or AKT or eNOS antibody. 
A

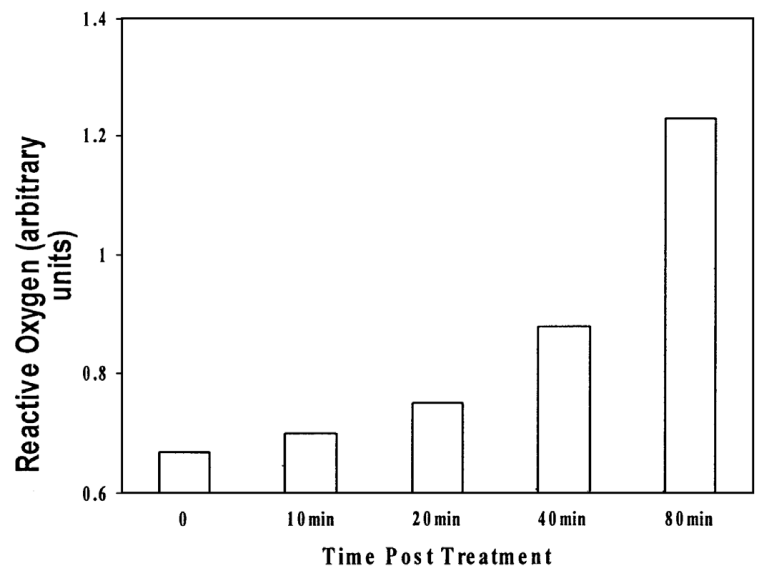

C
B

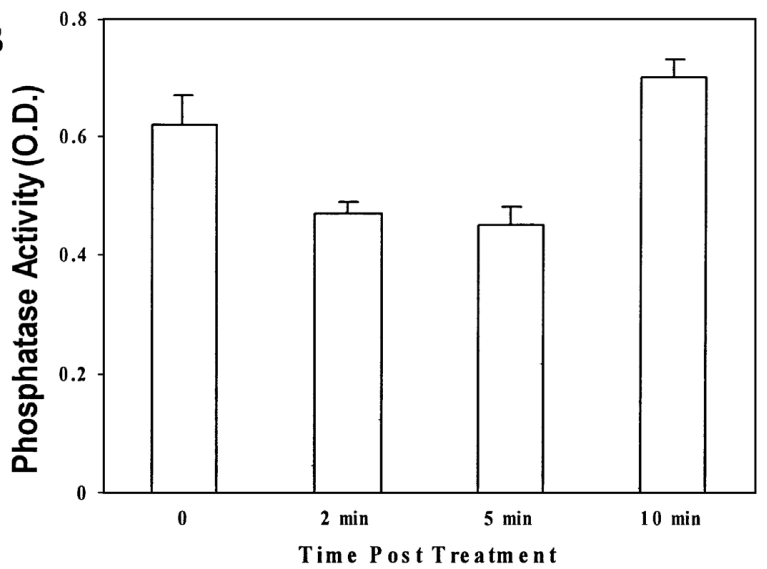

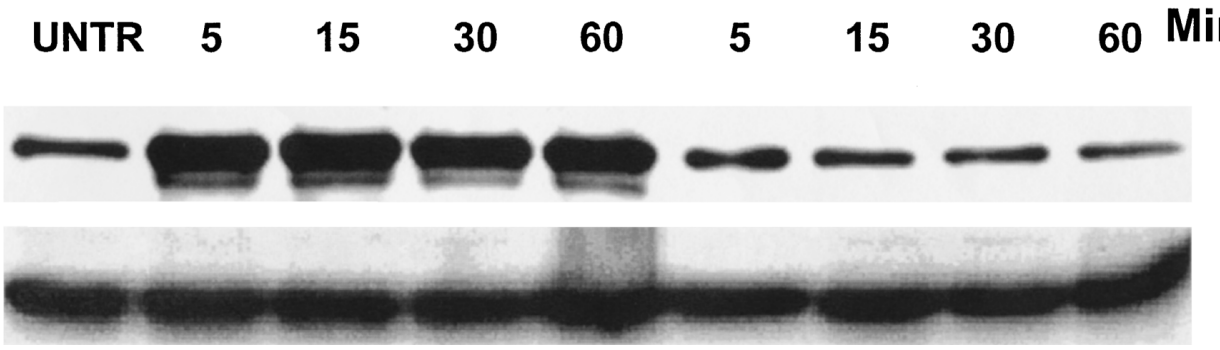 As $(200 \mu \mathrm{M})$ \\ EGF $(10 \mathrm{ng} / \mathrm{ml})$

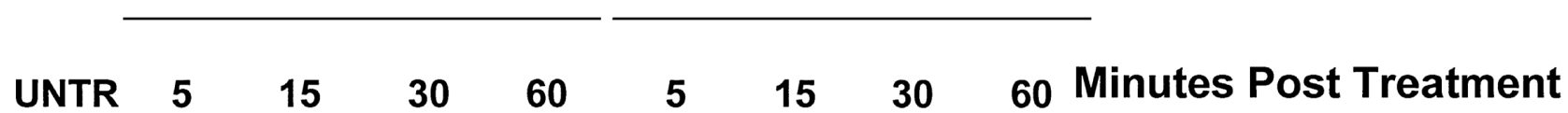 \\ Fig. 3. Arsenite induces ROS generation and inhibits phosphatase activity but does not activate EGFR. (A) Arsenite induces ROS generation. HaCat cells were loaded with $1 \mu \mathrm{M}$ of DHR for $1 \mathrm{~h}$, then treated with $200 \mu \mathrm{M}$ of sodium arsenite, and cells were fixed at various time points post treatment. ROS production was measured by FACS analysis. Three independent experiments were performed. Presented here was the result of a representative experiment. (B) Arsenite inhibits phosphatase activity in cultured human keratinocytes. Cultured human keratinocytes were treated with arsenite $(200 \mu \mathrm{M})$ and cells were collected at various time points. Phosphatase activity assay was performed as described in materials and methods. (C) Arsenite does not activate EGF receptor in cultured human keratinocytes. Cells were treated with EGF (100 ng/ml, as a positive control) or arsenite $(200 \mu \mathrm{M})$ and cells were collected at various time points. Proteins from cell extracts were subjected to immunoprecipitation with anti-EGFR antibody and SDS-PAGE and Western blot analysis. Phosphorylated- or total-EGFR was probed with anti-PY-20 or anti-EGFR antibody.}

Generation of ROS started 10 min post arsenite treatment, increased over time up to $80 \mathrm{~min}$. Recent data suggest that the balance between kinase and phosphatase activity account for the activation of cell surface components that may or may not be receptors. We next examined whether arsenite affects cellular phosphatase activity in human keratinocytes. The results showed that while arsenite inhibition of phosphatase activity started 2 min post treatment and peaked at $5 \mathrm{~min}$, the activity returned to normal within $10 \mathrm{~min}$ (Fig. 3B). This result indicates that arsenite does transiently inactivate phosphatase activity. It has been established that the activation of PI3K/AKT pathway is subsequent to the activation of cell growth factor receptor upon ligand binding (12). We found in our previous study however that UV irradiation activates this pathway following EGFR activation yet in a ligand independent manner (6). As we had observed in this study that arsenite induced the PI3K/AKT pathway, we further investigated whether arsenite induces EGF receptor activation in cultured human keratinocytes. As expected, EGF treatment induced tyrosine phosphorylation of EGFR in a time dependent manner, however, EGF receptor activation was not observed in response to arsenite exposure (Fig. 3C).

\section{Discussion}

Arsenite is a well-documented carcinogen. Various types of human cancers have been associated with exposure of arsenite. It has been reported that high level of arsenite in drinking water led to the increased risks of skin, bladder, kidney, lung and colon cancer $(1,2)$. While a complete understanding of the molecular mechanisms of arsenite-induced human cancer remains a daunting task, it is still of great interest to both scientists and laymen.

Recent study has suggested that activation of AKT in mammary epithelium provides a critical 
cell survival signal required for tumor progression (5). Phosphorylation of AKT by PI3K results in the inhibition of proapoptotic signals and the promotion of survival signals (7). We observed that arsenite activated AKT in cultured human keratinocytes in a time dependent manner. We also noticed that arsenite induced phosphorylation of eNOS, which is one of the known downstream components in the AKT pathway. eNOS is a member of the nitric oxide synthase family which also includes iNOS, and nNOS. Nitric oxide has been reportedly involved in vasodilation, inflammation and cytotoxicity (14). Over production of NO is associated with inflammation, neurodegenerative diseases, rheumatoid arthritis, diabetes mellitus, and DNA modification to cancer (11). Treatment with arsenite increased nitrate production, and Asinduced DNA strand breaks in bovine aortic endothelial cells could be decreased by nitrate oxide synthase inhibitors (10). Interestingly, our data indicated that $\mathrm{H}_{2} \mathrm{O}_{2}$ also induces activation of AKT and eNOS. That led us to believe that arsenite may induce ROS generation, which may be responsible for AKT and eNOS activation. Our data supported the possibility that arsenite induces the generation of ROS as shown previously by others (15). Surprisingly, however, in further studies using antioxidants NAC and PDTC, and the NADPH oxidase inhibitor DPI, we did not see the inhibition of AKT induced by arsenite. This suggests that the arseniteinduced AKT activation is not mediated by oxidants. Even more interestingly, we found that the p38 inhibitor SB203085 completely inhibited arsenite-induced activation of AKT, suggesting that in fact p38 mediates the arsenite-induced activation of AKT.

In response to growth factor stimulation activation of PI3K/AKT cell survival pathway starts with the activation of cell surface receptors $(12,16)$. It has been suggested that EGFR and Shc are critical mediators in the activation of the Ras/ERK signaling cascade by arsenite and arsenite may act as a tumor promoter largely by usurping this growth factor signaling pathway (17). However, in cultured human keratinocytes surprisingly we did not observe EGFR phosphorylation post arsenite exposure, although our data did not exclude the involvement of other cell surface receptors. It has been shown that arsenite treatment induces overexpression of growth factors in human keratinocytes (18). However, the time frame did not seem to match the time for the activation of AKT through surface receptors, because the mRNA levels of growth factors were detected four hours after arsenite treatment, before AKT activation. The overexpression of growth factors after arsenite exposure may be the result of activation of cell signal transduction pathways including the PI3K/AKT pathway. A recent model pointed out that the balance between kinases and phosphatases accounts for the cell surface receptor activation independent of ligand upon extracellular

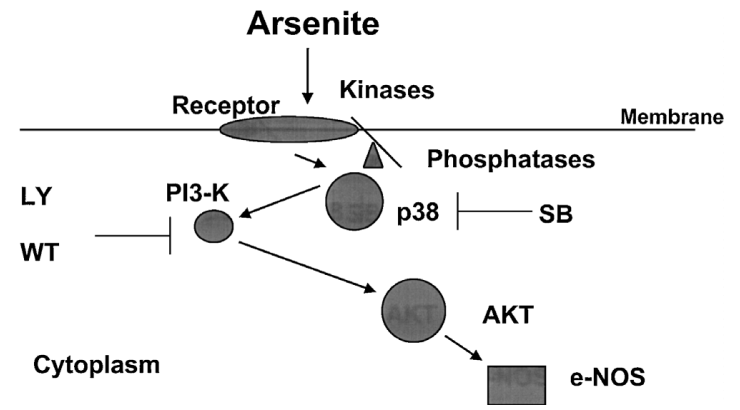

Fig. 4. Sequence of events upon arsenite exposure of human keratinocytes. Arsenite induces phosphorylation of AKT and eNOS that is mediated by PI3K and p38. Activation of PI3K and p 38 after arsenite treatment may be due to the imbalance between cellular kinases and phosphatases and the membranous activity that may or may not involve cell surface receptors.

stimuli (12). We observed that arsenite treatment does inactivate phosphatase activity in cultured human keratinocytes, these data agree with previously published evidence that arsenite inactivates JNK phosphatase specifically (19), and total cellular phosphatase activity in human fibroblasts (20).

In summary, we demonstrate that arsenite induces the activation of the PI3K/AKT cell survival pathway and that the PI3K/AKT pathway is mediated by $\mathrm{p} 38$ in cultured human keratinocytes. Arsenite activation of this pathway is independent of EGFR activation. The inhibition of phosphatase activity accounts for the altered balance between kinases and phosphatases which in turn likely contributes to the activation of cell surface components that may or may not be receptors (Fig. 4). The search for the membrane events is an ongoing project in the laboratory.

\section{Acknowledgments}

This study was supported in part by a grant for biomedical research from the Rhode Island Foundation, by a grant from the Committee on Aid to Faculty Research from Providence College, and by a grant from Avon Products, Inc.

\section{References}

1. Nriagu JO. (1994) Arsenic in the environment. II. Human Health and Ecosystem Effects. Wiley and Sons, New York.

2. Smith AH, Hopenhayn-Rich C, Bates MN, et al. (1992) Cancer risks from arsenic in drinking water. Environ. Health Perspect. 97: 259-267.

3. Trouba KJ, Wauson EM, Vorce RL. (2000) Sodium arseniteinduced dysregulation of protiens involved in proleferative signaling. Toxicol. Appl. Pharmacol. 164: 161-170.

4. Hamadeh HK, Vargas M, Lee E, Menzel DB. (1999) Arsenic disrupts cellular levels of $\mathrm{p} 53$ and mdm2: a potential mechanism of carcinogenesis. Biochem. Biophys. Res. Commun. 263: 446-449.

5. Hutchinson J, Jin J, Cardiff RD, et al. (2001) Activation of Akt (protein kinase B) in mammary epithelium provides a critical cell survival signal required for tumor progression. Mol. Cell. Biol. 21: 2203-2212. 
6. Wan YS, Wang ZQ, Shao Y, et al. (2001) UVB activates PI-3 kinase/PKB pathway in cultured keratinocytes in vitro and human skin in vivo. Int. J. Oncology 18: 461-466.

7. Kops GJ, de Ruiter ND, De Vries-Smits AM, et al. (1999) Direct control of the Forkhead transcription factor AFX by protein kinase B. Nature 398: 630-634.

8. Wan YS, Wang ZQ, Shao Y, et al. (2000) UV-induced expression of GADD45 is mediated by an oxidant sensitive pathway in cultured human keratinocytes and human skin in vivo. Int. J. Mol. Med. 6: 683-688.

9. Huang C, Ma MY, Li J, Dong Z. (1999) Arsenic induces apoptosis through a c-Jun NH2-terminal kinase-dependent, p53independent pathway. Cancer Res. 59: 3053-3058.

10. Liu SX, Athar M, Lippai I, et al. (2001) Induction of oxyradicals by arsenic: Implication of mechanism of genotoxicity. Proc. Natl. Acad. Sci. USA 98: 1643-1648.

11. Fulton D, Gratton J, McCabe TJ, et al. (1999) Regulation of endothelium-derived nitric oxide production by the protein kinase Akt. Nature 399: 597-600.

12. Franke TF, Kaplan DR, Cantley LC. (1997) PI3K: Downstream AKTion Blocks Apoptosis. Cell 88: 435-437.

13. Suzuki YJ, Forman HJ, Sevanian A. (1997) Oxidants as stimulators of signal transduction. Free Radical Biology $\theta$ Medicine 22: 269-285.
14. Lowenstein CJ, Snyder SH. (1992) Nitric oxide: a novel biological messenger. Cell 70: 705-707.

15. Hei TK, Liu SX, Waldren, C. (1998) Mutagenicity of arsenic in mammalian cells: role of reactive oxygen species. Proc. Natl. Acad. Sci. USA 95: 8103-8107.

16. Wang XT, McCullough KD, Franke TF, Holbrook NJ. (2001) Epidermal growth factor receptor-dependent AKT activation by oxidative stress enhanced cell survival. J. Biol. Chem. 275: 14624-14631.

17. Chen W, Martindale JL, Holbrook JN, Liu Y. (1998) Tumor promoter arsenite activates extracellular signal-regulated kinase through a signaling pathway mediated by epidermal growth factor receptor and shc. Mol. Cell. Biol. 18: 51785188.

18. Germolec DR, Spalding J, Yu HS, et al. (1998) Arsenic enhancement of skin neoplasia by chronic stimulation of growth factors. Am. J. Pathol. 153: 1775-1785.

19. Cavigelli M, Li WW, Lin A, et al. (1996) The tumor promoter arsenite stimulates AP-1 activity by inhibiting a JNK phosphatase. EMBO J. 15: 6269-6279.

20. Huang RN, Ho IC, Yih LH, Lee TC. (1995) Sodium arsenite induces chromosome endoreduplication and inhibits protein phosphatase activity in human fibroblasts. Environ. Mol. Muta gen. 25: 188-196. 